\title{
Bridging Between Material Properties Of Proteins And The Underlying Molecular Interactions
}

\author{
Guang Song ${ }^{1,2 *}$ \\ ${ }^{1}$ Department of Computer Science, \\ ${ }^{2}$ Program of Bioinformatics and Computational Biology, \\ Iowa State University, Ames, IA 50011, USA \\ ${ }^{*}$ Correspondence to: Guang Song, Tel: 515-294-1696; \\ Fax: 515-294-0258; E-mail: gsong@iastate.edu
}

\begin{abstract}
In this work, we develop a novel method that bridges between material properties of proteins, particularly the modulus of elasticity, and the underlying molecular interactions. To this end, we employ both an all-atom normal mode analysis (NMA) model with the CHARMM force field and an elastic solid model for proteins and protein interfaces. And the "bridge" between the two models is a common physical property that they both can predict: the magnitude of thermal vibrations. This connection allows one to calibrate the Young's moduli of proteins and protein interface regions. We find that the elastic moduli of proteins are mostly in the range of a few Gpa to $10 \mathrm{Gpa}$, while the elastic moduli of the interface regions are about an order smaller. The work
\end{abstract}


is significant as it represents the first attempt to systematically compute the moduli of elasticity of proteins from molecular interactions.

Keywords: Stiffness; Young's modulus; elastic solid model; normal mode analysis; B-factor; proteins; molecular interactions

\section{Introduction}

Along with liquid, gas, and plasma, solid is one of the four states of matter. Atoms in solids are packed tightly and kept together by physical interactions, such as ionic bonds (as in sodium chloride), covalent bonds (as in diamond), metalic bonds (as in metals), hydrogen bonds (as in ice), or van der Waals interactions (as in organic compounds) ${ }^{1}$. Solids held together mostly by van der Waals interactions are called van der Waals solids.

The type of force that keeps a solid together determines its material properties, such as elasticity. Solids held together by metalic, ionic bonds, or covalent bonds appear stiff and have a much higher Young's moduli than solids held together mostly by van der Waals interactions such as proteins ${ }^{2}$.

The Young's moduli of proteins have been studied in three primary ways, namely, protein fibers or fibrils ${ }^{3}$, protein crystals ${ }^{4}$, and protein capsids ${ }^{5}$.

Among protein fibers, some exhibit high extensibility and have a very low Young's modulus, on the order of only 1-10 MPa. These fibers usually contain unstructured regions or high mobility motifs, undergo $\alpha$-helix to $\beta$-strand transitions, or even unfold under external forces, and consequently they appear very soft $(1-10 \mathrm{Mpa})^{3}$. Other protein fibers are significantly different as they "form highly regular, nearly crystalline arrangement of monomer units without regions that can extend, change secondary structure or unfold" ${ }^{3}$ and thus appear much stiffer. Experimental studies showed these fibers, such as actin ${ }^{6}$, tubulin, collagen $^{2}$, and keratin ${ }^{7}$, fibrin ${ }^{3}, \beta$-lactoglobulin fibrils ${ }^{8}$, had an elastic modulus in the range 
of a few Gpa. The Young's moduli of these fiber proteins were obtained through direct measurement of force under stretching, mostly using atomic force spectroscopy (AFM $)^{8,9}$.

The mechanical properties of proteins were studied also using protein crystals, mostly the crystals of hen egg-white lysozyme (HEWL), a model protein. Table 1 summarizes the techniques used, proteins studied, and results obtained. It lists also results from the compressibility studies done in solvent ${ }^{10}$ and on a crystal structure solved at high pressure $(1,000 \text { bar })^{11}$. The results show a large variation, from less than 1 Gpa to over 10 Gpa. Compressibility study by Gavish et al. ${ }^{10}$ showed that under most conditions, the adiabatic compressibilities of lysozyme, hemoglobin, and myoglobin in solution were on the order of $10-20 \%$ of the compressibility of water, or $5-7 \times 10^{-11} \mathrm{~m}^{2} / N$. Consequently, the bulk moduli of these proteins, which are the inverse of their compressibilities, are over 10 Gpa. Kundrot and Richards ${ }^{11}$ found that the isothermal compressibility of lysozyme proteins was $4.710^{-3}$ $\mathrm{kbar}^{-1}$, or 20 Gpa in bulk modulus. On the other hand, the Young's moduli obtained from protein crystals using techniques such as vibration, indentation, ultrasound, or Brillouin scattering are much smaller, from 0.3 Gpa to 5.5 Gpa (see Table 1). How to understand this discrepancy? It is possible that the larger values obtained from compressibility studies represent the elastic modulus of proteins themselves, while the elastic moduli of protein crystals probably represent the Young's moduli of both proteins and protein interface regions, as well as other noncrystalline elements in the crystal cell such as intracrystalline liquid ${ }^{4}$. This would reconcile the seemingly conflicting results on Young's moduli of proteins in the literature.

The Young's moduli of proteins measured experimentally as reviewed above depend on a number of factors, including conditions of the samples (such as $\mathrm{pH}$ in solvent ${ }^{10}$ or water content in crystals, i.e., moistened or dried ${ }^{15}$, etc.), and the frequency range at which a measurement was carried out ${ }^{17}$, which varies from static to kilohertz ${ }^{12-14}$, to ultrasonic in the megahertz range ${ }^{16,17}$ and Brillouin light scattering method in the gigahertz range ${ }^{18}$. 
Table 1: A summary of elastic modulus studies using protein crystals and bulk modulus studies based on compressibility measured in solvent or in crystal under high pressure.

\begin{tabular}{|l|r|r|r|}
\hline Techniques & Proteins & Young's modulus & References \\
\hline vibrating reed & lysozyme & $0.3-1.5 \mathrm{Gpa}$ & {$[12-14]$} \\
indentation & lysozyme & $0.49-4.2 \mathrm{Gpa}$ & {$[15]$} \\
ultrasound & lysozyme & $4.87-5.5 \mathrm{Gpa}$ & {$[16,17]$} \\
brillouin scattering & lysozyme & $5.5 \mathrm{Gpa}$ & {$[18]$} \\
\hline & & bulk modulus & \\
\hline ultrasound in solution & lysozyme, Mb, Hb & $>10 \mathrm{Gpa}$ & {$[10]$} \\
X-ray at high pressure & lysozyme & $>20 \mathrm{Gpa}$ & {$[11]$} \\
\hline
\end{tabular}

The Young' modulus of a protein is also temperature dependent. Morozov and Gevorkian analyzed that the mechanical properties of protein crystals at different temperature and found that proteins became significantly more rigid below glass transition temperature, when the surface layer of proteins and their bound water became immobilized ${ }^{4}$. In this work, we also will investigate the influence of temperature on a protein's modulus.

The elastic modulus of proteins were studied also through protein capsids by nanoindentation using atomic force microscopy (AFM). Nano-indentation allows one to measure the stiffness of viral capsids. The elastic modulus of the capsid material can then be deduced from the measured stiffness. However, in order to do so, in most cases a thin-shell model was assumed and thin-shell elasticity theory applied. The limitation of the thin shell model is that it neglects the molecular structure of the capsid and assumes a homogeneous material property throughout, as well as an idealized spherical geometry and uniform thickness ${ }^{5}$. Thick shell models combined with finite element analysis were also tried ${ }^{19}$ and the effect of non-uniform geometry was investigated ${ }^{20}$. These work all represent a top-down approach since experimental determined stiffness values were used in all cases to fit the underlying elastic moduli of capsids. A review article in 2012 by Mateu ${ }^{5}$ summarized the nano-indentation results of a dozen viral capsids: the stiffness of the viral capsids and the derived Young's moduli. The review showed the Young's moduli of capsid proteins varied over a span of more than one order of magnitude, from 0.14 MPa for CCMV to 1-3 GPa for MVM and $\Phi 29^{5}$. The 
range of variation in Young's moduli seen in capsids is consistent with what is seen in protein crystals (Table 1, top half) but is much lower than what are obtained from compressibility studies (Table 1, bottom half), A possible explanation is that Young's moduli thus obtained represent the average moduli of both proteins and protein interface regions, while the elastic moduli obtained from compressibility studies represent those of individual proteins.

The Young's moduli of globular proteins were also estimated theoretically or computationally. By approximating proteins as solid materials with sheets that interacted through van der Waals interactions, Howard estimated that the Young's modulus of proteins to be around 4 Gpa (see Appendix 3.1 of 2). In the case of F-actin, Bathe applied axial stretching of his elastic solid model for actin to match experimental stretching stiffness data ${ }^{6}$ and found that the effective Young's modulus of actin was $2.7 \mathrm{Gpa}^{21}$.

Contribution of this work. In this work, we develop a novel bottom-up approach for computing the elastic moduli of proteins by bridging between the material properties of proteins with their underlying molecular interactions described by the accurate CHARMM force field. Our approach utilizes both normal mode analysis (NMA) ${ }^{22-24}$, a well established technique for studying the fluctuating dynamics of macromolecules, and elastic solid models $(\mathrm{ESM})^{25}$ developed more recently that treat macromolecules as elastic solids with material properties such as Young's modulus. Since both of these models can predict the magnitude of thermal vibrations of macromolecules, this commonality is used as a bridge to link material properties modeled in $\mathrm{ESM}^{25}$ with molecule interactions used in NMA. To the best of our knowledge, This work represents the first attempt to determine the Young's moduli of proteins and protein interface regions separately and systematically from all-atom molecular interactions. We find the Young's modulus of proteins can be as high as $10 \mathrm{Gpa}$, while the Young's modulus of protein interface regions is an order smaller. Our work reconciles the high modulus values found through compressibility studies and low values found in protein crystals or capsids. The large span of variations of elastic moduli at interface regions pro- 
vides an explanation also for the similar extent of variations seen in protein crystals and protein capsids.

\section{Methods}

\section{$2.1 \quad \sigma$ ESM, a molecular surface-based elastic solid model}

Recently, we presented a novel elastic solid model called $\alpha \mathrm{ESM}^{25}$ for macromolecules based on alpha shape ${ }^{26}$. The model has a parameter alpha which was chosen empirically.

Here we present a new extension of $\alpha \mathrm{ESM}$ whose major improvement over $\alpha \mathrm{ESM}$ is that the alpha value is now tuned individually for each protein so that the final elastic solid model of the protein has the same volume as its solvent excluded volume, such as that computed by the MSMS algorithm ${ }^{27}$. Another significant difference of the present ESM model from $\alpha$ ESM is that in representing the solid, not only the atomic coordinates of the protein are used, which is the case for $\alpha$ ESM, but also selected points on the protein's molecular surface ${ }^{21}$. Consequently, the final solid model also has a similar surface to the molecular surface of the protein. We name this new variant of $\mathrm{ESM} \sigma \mathrm{ESM}$, since $\sigma$ is a symbol commonly used for surface area or surface tension ${ }^{28}$.

The procedural flow of $\sigma$ ESM is given in Fig. 1. The script takes as input $x y z$, which contains the coordinates of all the heavy atoms of a given protein structure that is available in its PDB file, and verts, faces, and volume, all three of which are outputs of the MSMS software and represent respectively the vertices and faces of the solvent excluded surface mesh and its enclosed volume (line 1). The program first computes an initial alpha shape of the protein alone (line 11), which is used to fix the number of faces that should be on the surface mesh (lines 12-13). It then simplifies the surface mesh produced by MSMS using MATLAB script reducepatch (line 15). The vertices on the reduced molecular surface are then appended to the coordinates of the atoms in the protein (line 16). Lastly, an alpha 
value is selected (line 18) so that the final alpha shape has the same volume as the the volume computed from MSMS ${ }^{27}$. Once the alpha value is determined, the script used for $\alpha$ ESM can be adopted to compute the stiffness matrix $\mathbf{K}$ and mass matrix $\mathbf{M}$ (line 19), from which eigenmodes and eigen frequencies can be computed (Fig. 1 in Ref. 25).

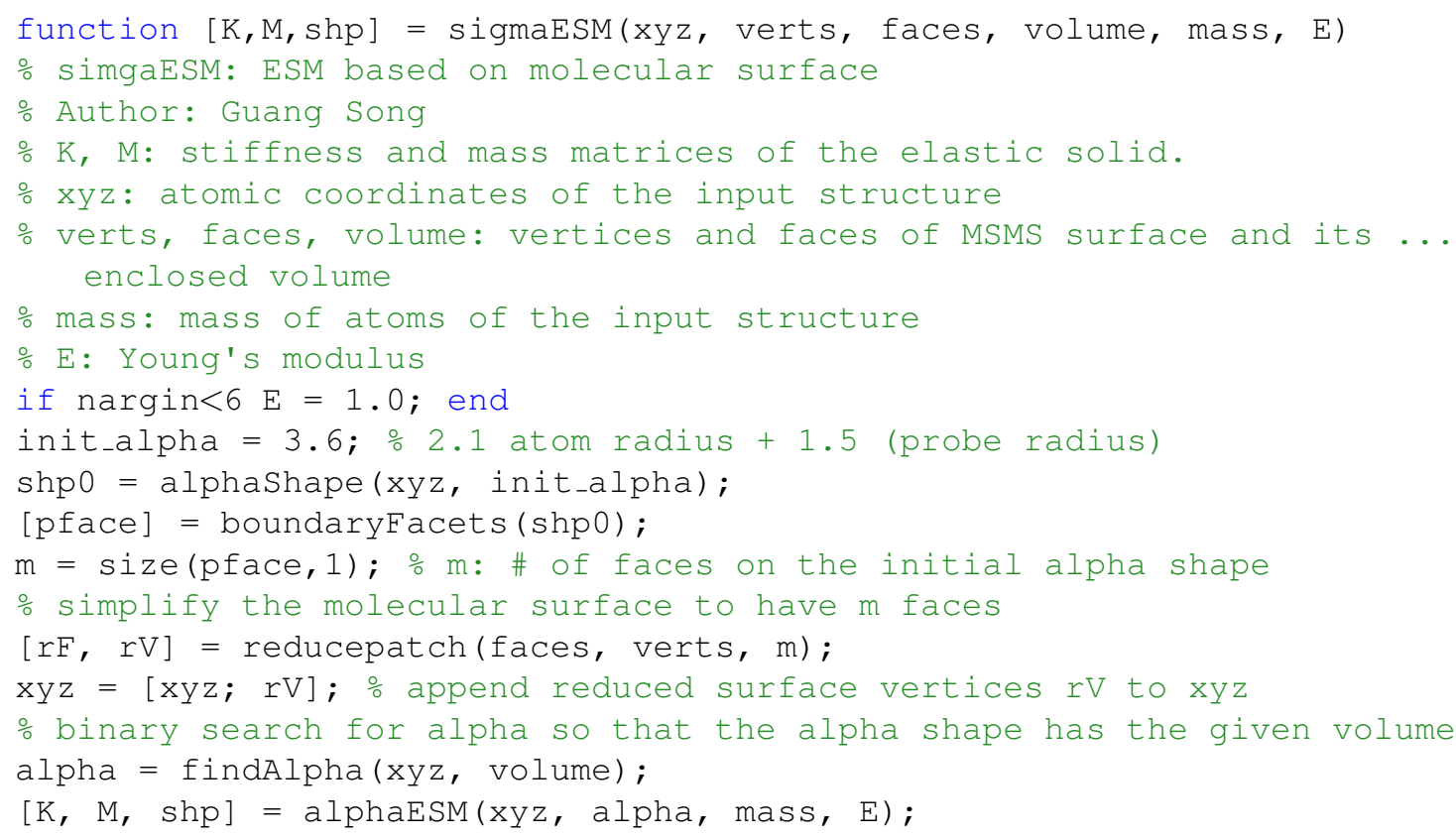

Figure 1: The algorithmic flow of $\sigma$ ESM as expressed in a MATLAB script. A complete copy of the above MATLAB script is available at https://github.com/gsongISU/sigmaESMrelease. The scripts for computing stiffness matrix and mass matrix used in alphaESM are available at MATLAB file exchange (https://www.mathworks.com/matlabcentral/fileexchange/ 27826-fast-fem-assembly-nodal-elements), kindly contributed by Anjam and Vald$\operatorname{man}^{29}$.

Fig. 2 shows the $\alpha$ ESM model of a protein (pdb-id: 1aqb) in red. The molecular surface, or solvent excluded surface, computed from the MSMS software ${ }^{27}$ is shown in blue as a triangular surface mesh. The total volume of the $\alpha$ ESM solid model (shown in red) is 16,600 $\AA^{3}$, while the solvent excluded volume is $23,720 \AA^{3}$. Figure $2(\mathrm{~B})$ shows the $\sigma$ ESM model in cyan, which has an extra layer over the $\alpha$ ESM model and resembles closely the molecular 
surface in Figure 2(A). Specifically, the volume of $\sigma$ ESM model is $23,770 \AA^{3}$, nearly the same as the protein's solvent excluded volume.

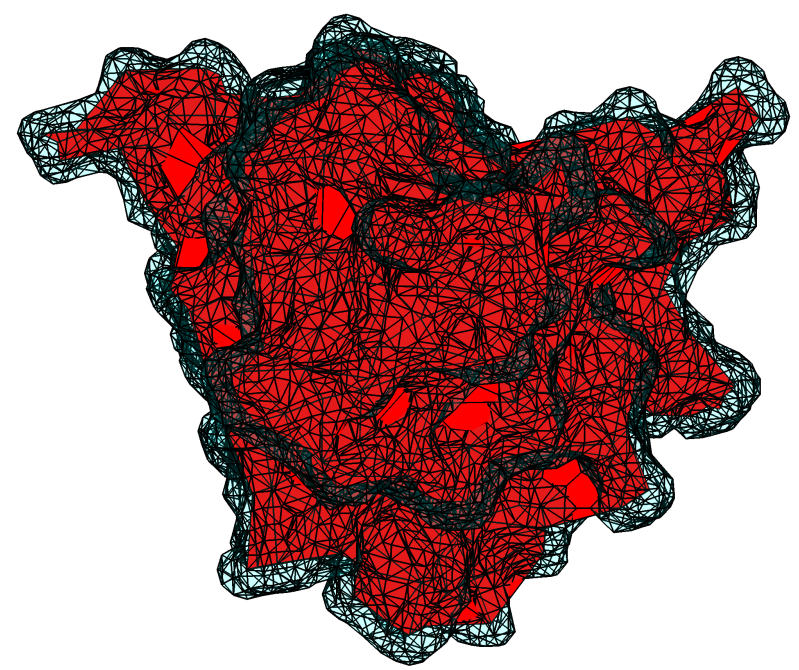

(A)

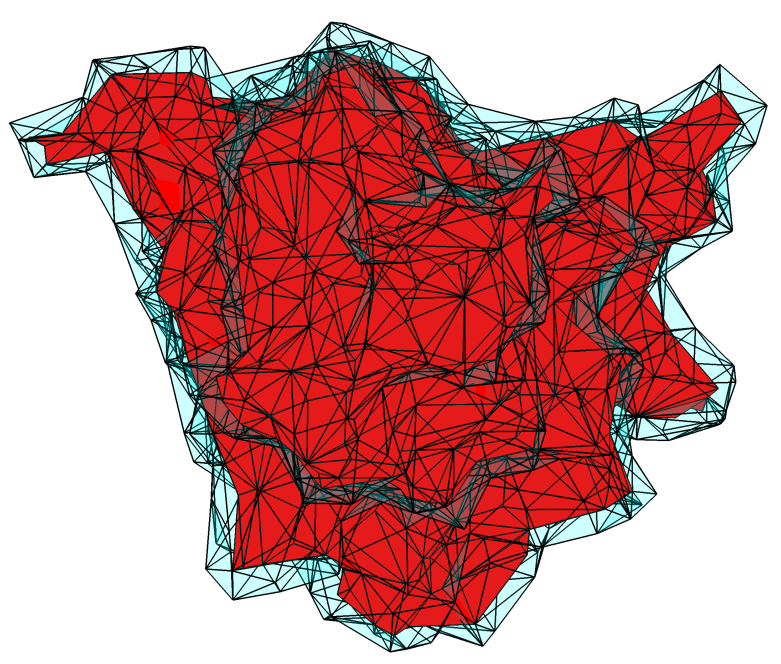

(B)

Figure 2: The $\alpha \mathrm{ESM}_{\text {model }}{ }^{25}$ (in red) using alpha $=3.6 \mathrm{~A}$. The solvent excluded surface computed from $\mathrm{MSMS}^{27}$ is shown in blue as a triangle mesh. (B) The $\sigma \mathrm{ESM}$ model: the difference between it and $\alpha \mathrm{ESM}$ (in red) is shown in cyan.

In summary, $\sigma \mathrm{ESM}$ is a more accurate representation than $\alpha \mathrm{ESM}$ in describing both molecular surface and and its enclosing volume.

The magnitude of thermal vibrations by $\sigma$ ESM. Given a protein structure and its molecular surface mesh (which can be generated for instance by the MSMS software ${ }^{27}$ ), an application of the $\sigma$ ESM script given in Fig. 1 produces a stiffness $(\mathbf{K})$ and a mass $(\mathbf{M})$ matrix. For the mass matrix used in finite element method, one has the option of a consistent mass matrix or a lump-sum mass matrix ${ }^{30}$. In this work, the lump-sum mass matrix is used, which is the same as the mass matrix used in NMA and is diagonal. (The consistent mass matrix, on the other hand, is not a diagonal matrix.) According to Ref. 25, the mean-square 
thermal vibrations of atom $i$ by ESM is:

$$
\left\langle\Delta \mathbf{R}_{i}^{2}\right\rangle_{\mathrm{ESM}}=\frac{k_{B} T}{E} \operatorname{trace}\left(\left[\mathbf{M}^{-\frac{1}{2}} \mathbf{K}_{m}^{-1} \mathbf{M}^{-\frac{1}{2}}\right]_{i i}\right)
$$

where the subscript $i i$ represents the $\mathrm{i}^{\text {th }} 3$-by-3 diagonal block and $\mathbf{K}_{m}$ is the mass-weighted stiffness matrix, i.e., $\mathbf{K}_{m}=\mathbf{M}^{-1 / 2} \mathbf{K M}^{-1 / 2}$.

The magnitudes of thermal vibrations of a whole protein is defined as the mass-weighted averages of $\left\langle\Delta \mathbf{R}_{i}^{2}\right\rangle_{\mathrm{ESM}}$ over all the nodes ${ }^{31}$, i.e.,

$$
\left\langle\Delta \mathbf{R}^{2}\right\rangle_{\mathrm{ESM}}=\frac{\sum_{i} m_{i}\left\langle\Delta \mathbf{R}_{i}^{2}\right\rangle_{\mathrm{ESM}}}{\sum_{i} m_{i}}=\frac{k_{B} \operatorname{Ttrace}\left(\mathbf{K}_{m}^{-1}\right)}{E \sum_{i} m_{i}},
$$

where $i$ is the index of nodes, which include both protein atoms and added molecular surface nodes. $m_{i}$ are the mass of node $i$. Each surface node is given a mass of 1 atomic unit.

\subsection{The sbNMA model}

The sbNMA model developed by $\mathrm{Na}$ and $\mathrm{Song}^{32}$ is an all-atom NMA model based on the CHARMM force field ${ }^{33}$. It is designed to maintain the high accuracy of classical NMA (cNMA) using all-atom force fields. By circumventing the cumbersome step of energy minimization, it can be applied directly to experimental structures. Extensive studies have demonstrated its accuracy ${ }^{31,32,34-36}$. The entire sbNMA code is publicly available at https://github.com/htna/sbNMA-Matlab. Given a protein structure, an application of the sbNMA code (particularly the sbNMA_PSF.m script available at the above link) produces a Hessian matrix $\mathbf{H}$ and a mass matrix $\mathbf{M}$ that is diagonal (the sbNMA_PSF script actually produces the diagonal of the mass matrix as a vector, which can be easily converted to a mass matrix when needed).

Once the Hessian matrix $\mathbf{H}$ and the mass matrix $\mathbf{M}$ are obtained, the mean square 
fluctuation of atom $i$ is ${ }^{32}$ :

$$
\left\langle\Delta \mathbf{R}_{i}^{2}\right\rangle_{\mathrm{NMA}}=k_{B} \operatorname{Ttrace}\left(\left[\mathbf{M}^{-\frac{1}{2}} \mathbf{H}_{m}^{-1} \mathbf{M}^{-\frac{1}{2}}\right]_{i i}\right)
$$

Thus,

$$
\left\langle\Delta \mathbf{R}^{2}\right\rangle_{\mathrm{NMA}}=\frac{\sum_{i} m_{i}\left\langle\Delta \mathbf{R}_{i}^{2}\right\rangle_{\mathrm{NMA}}}{\sum_{i} m_{i}}=\frac{k_{B} \operatorname{Ttrace}\left(\mathbf{H}_{m}^{-1}\right)}{\sum_{i} m_{i}}
$$

As will be shown later, the magnitude of thermal vibrations as computed from sbNMA in Eq. (4) can be used to calibrate the Young's moduli of proteins.

\subsection{B-factors, static disorder, and glass transition}

The Debye-Waller factor (DWF), or B-factor as it is called in protein X-ray crystallography, is a factor used to describe for each atom the degree to which electron density spreads out. The spread around the mean position of each atom $i$ is generally modeled as a Gaussian function and the magnitude of the spread is characterized by $\left\langle u_{i}^{2}\right\rangle$, the mean-square displacement from the mean position. The isotropic Debye-Waller factor, or B-factor, is related to $\left\langle u_{i}^{2}\right\rangle$ as:

$$
\left\langle u_{i}^{2}\right\rangle=\frac{3}{8 \pi^{2}} B_{i}
$$

The magnitude of mean-square displacement of a whole protein is defined as the massweighted averages of $\left\langle u_{i}^{2}\right\rangle$ over all the atoms ${ }^{31}$, i.e.,

$$
\left\langle u^{2}\right\rangle=\frac{\sum_{i} m_{i}\left\langle u_{i}^{2}\right\rangle}{\sum_{i} m_{i}}
$$

where $i$ is the atom index. 


\subsection{Determine the Young's moduli of proteins based on molecular interactions}

sbNMA is an accurate NMA model for predicting the magnitude of thermal vibrations. However, like all NMA models and elastic network models, one major limitation of sbNMA is that it assumes harmonicity. Thus the predicted magnitude by sbNMA represents only a lower bound of the actual magnitude of fluctuations of proteins that may contain also anharmonic motions, especially at above $T_{g}$, the temperature of glass transition ${ }^{37}$.

The magnitude of thermal vibrations can be obtained also experimentally. X-ray crystallography that is widely used in protein structure determination produces also dynamical information in so-called B-factors. Since B-factors contain also a significant amount of static disorder ${ }^{31}$, the magnitude of mean-square displacement obtained from B-factors thus represents an upper bound of the actual magnitude of fluctuations of proteins.

A possible third alternative for obtaining the magnitude of thermal fluctuations of proteins is molecular dynamics (MD) simulation ${ }^{38,39}$, which is able to take into account anharmonic motions and is unaffected by static disorder. However, it suffers from the problem of insufficient sampling of the conformation space. Consequently, the actual magnitude of thermal fluctuations is difficult to obtain using MD.

The Young's modulus represents a material property of a solid. By representing a protein using both a molecular model (as in NMA) and an elastic solid model as in $\sigma$ ESM, we can link material property with molecular interactions. Particularly, we can deduce a protein's Young's modulus by requiring the magnitude of thermal vibrations predicted by $\sigma \mathrm{ESM}$ in Eq. (2) to be the same as the magnitude obtained from sbNMA in Eq. (4), or the magnitude of mean-square displacement from B-factors in Eq. (6). Since the magnitudes obtained from sbNMA and B-factors represent the lower and upper bounds of the actual magnitude respectively, the Young's moduli thus deduced represent the upper and lower bounds of the 
actual Young's moduli. Specifically, by requiring $\left\langle\Delta \mathbf{R}^{2}\right\rangle_{\mathrm{ESM}}=\left\langle\Delta \mathbf{R}^{2}\right\rangle_{\mathrm{NMA}}$ (eqs. (2) and (4)), we have:

$$
E_{\text {upper }}=\frac{\operatorname{trace}\left(\mathbf{K}_{m}^{-1}\right) / m_{t o t}^{\mathrm{ESM}}}{\operatorname{trace}\left(\mathbf{H}_{m}^{-1}\right) / m_{\text {tot }}^{\mathrm{NMA}}}
$$

where $m_{t o t}^{\mathrm{ESM}}$ and $m_{\text {tot }}^{\mathrm{NMA}}$ are the total masses in $\sigma$ ESM and NMA, respectively. And similarly, by requiring $\left\langle\Delta \mathbf{R}^{2}\right\rangle_{\mathrm{ESM}}=\left\langle u^{2}\right\rangle$ (eqs. (2) and (6)), we have:

$$
E_{\text {lower }}=\frac{\operatorname{trace}\left(\mathbf{K}_{m}^{-1}\right) / m_{t o t}^{\mathrm{ESM}}}{\left(\sum_{i} m_{i}\left\langle u_{i}^{2}\right\rangle\right) / m_{t o t}^{\text {Bfac }}}
$$

where $m_{t o t}^{B f a c}$ is the total mass of all the atoms with B-factors, which usually are the heavy atoms.

\subsection{Quaternary Structures based on crystal contacts}

For each protein chain $p$ in the dataset, we determine the all the other chains in its unit cell based on the space group and symmetry matrices given in its PDB file. Additionally we find all the neighboring cells to the current cell in all directions: altogether $27-1=26$ neighboring cells are considered. Of all the chains in these cells, we identify the one that is the closest to $p$ and denote this chain as $q . p$ and $q$ form a crystal contact and a quaternary structure. (In the case when the selected $q$ has a very small contact surface with $p$, a different chain $q$ is used instead.) The quaternary structures are saved in PDB files as xxxx2.pdb, where xxxx is the pdb-id, and are available at https://github.com/gsongISU/sigmaESMrelease, under folder pdbDataset. In the following, we explain how to compute the Young's modulus of the interface region of a quaternary structure. 


\section{6 $\sigma \mathrm{ESM}$ of the quaternary structures}

$\sigma \mathrm{ESM}$ is readily applicable to the aforementioned quaternary structures in the same fashion as to regular tertiary structures, and as a result, an elastic solid model is produced (see Fig. 3 of structure 2pwa2.pdb) . The nodes in the model consists of heavy atoms of two protein chains (regions in red) in the quaternary structure and the surface nodes (in cyan) given by the MSMS software ${ }^{27}$. The elements in the solid model are tetrahedra generated by alpha shape $^{26}$ (see Fig. 1). Given the elastic solid model of a quaternary structure, the interface region, which is in blue in Fig. 3, is identified as follows.

First, all the tetrahedral elements formed by atoms from chain $p$ or surface nodes but not from chain $q$ are identified. Let all the nodes of these elements be nodes 1 . nodes 1 consists of all the atoms of chain $p$ and some surface nodes, but no atoms from chain $q$. Similarly, compute nodes 2 for the second chain $q$. Next, remove any common surface nodes shared by nodes 1 and nodes 2 from them. As a result, the intersection of nodes 1 and nodes 2 is an empty set. Finally, identify all the tetrahedral elements formed solely by nodes 1 or nodes 2. The remaining elements are identified as the interface region (in blue). 
bioRxiv preprint doi: https://doi.org/10.1101/2021.02.03.429521; this version posted February 3, 2021. The copyright holder for this preprint (which was not certified by peer review) is the author/funder, who has granted bioRxiv a license to display the preprint in perpetuity. It is made available under aCC-BY 4.0 International license.
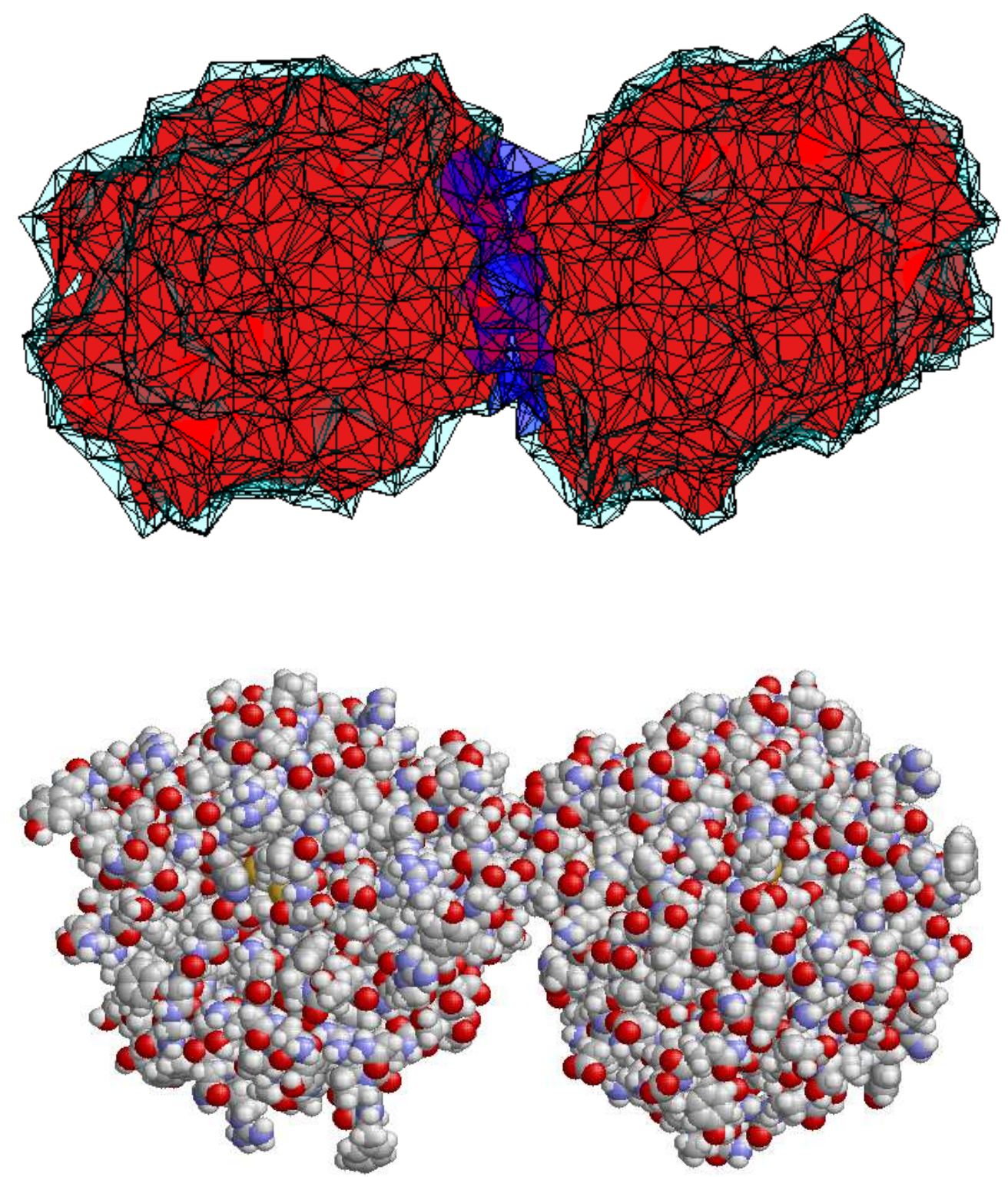

Figure 3: Models of a quaternary structure. (Top) An elastic solid model of a quaternary structure (2pwa2.pdb). The pdb quaternary structure is available at https://github.com/gsongISU/ sigmaESMrelease, under folder pdbDataset. (Bottom) the all-atomic molecular model of the same structure. The image is generated by Rasmol ${ }^{40}$. 


\subsection{Computing the Young's modulus of the interface region}

Next, we compute the Young's modulus of the interface region of the quaternary structure. This allows us to assess how "soft" or "firm" the region is. Again, our method to determine the material property of the interface region is to link it with the underlying molecular interactions using $\sigma$ ESM and sbNMA, by requiring the two models to give the same magnitude of thermal vibrations.

Fig. 3(B) shows the molecular structure model of the same quaternary structure after protonation using psfgen from $\mathrm{VMD}^{41}$. We apply sbNMA to compute its magnitude of the thermal vibrations using Eq. (4). To focus on the interface region and to speed up the computations, we set the two proteins as rigid, i.e., the internal degrees of freedom within each protein chain is left out, by employing a projection matrix as was done in the RTB model $^{42}$. The magnitude of thermal vibration of the whole structure (Fig. 3(B)) is thus dictated solely by inter-protein interactions. Similarly, when applying $\sigma$ ESM to the solid model in Fig. 3(A), the two protein regions, which are in red, are set as rigid and only the interface region (in blue) is flexible. By requiring the two models to give the same magnitude of thermal vibration, we can determine the Young's modulus of the interface region, similar to what is done in Eq. (7). The entire program for computing the Young's modulus of the interface region is given at https://github.com/gsongISU/sigmaESMrelease. The program uses $\sigma$ ESM and sbNMA and a matrix projection module. All of them are available at the above website.

It is worth noting that only non-bonded interactions (possibly also disulfide bonds) are present at the interface region. Therefore it is expected that the Young's modulus of the interface region should be smaller than that of a protein itself, since the latter contains many bonded interactions. Also, quaternary structures constructed from crystal contacts as described above, especially after protonation, may contain steric clashing in the interface region. For atom pairs that are too close to each other (i.e., closer than their equilibrium 
separation distance which is the sum of their van der Waals radii), we use their equilibrium separation distances as their separation distances when computing the potential energy at the interface.

\section{Results}

\subsection{The Choice of Protein Dataset}

In our recent work ${ }^{31}$, we have shown that the amount of thermal vibrations is small in most proteins but is significantly large (50\% or higher) for structures determined at both high resolution and high temperature (that is, room temperature). Unfortunately such structures are rare in the PDB, as most structures in the PDB are determined at cryogenic temperature to reduce the radiation damage ${ }^{43}$.

Based on this premise, we select a group of 18 structures determined at high resolution (1.1 $\AA$ or high) and at room temperature. This set of structures are taken from a list of 1522 structures used in our previous work ${ }^{31}$ that was originally generated by PDB_SELECT ${ }^{44,45}$.

\subsection{Upper bound and lower bound of thermal fluctuations in pro- teins}

There are a few universal properties of globular proteins that are intriguing. First, it was established that the vibrational spectra of globular proteins, once properly normalized, follow a universal curve ${ }^{34,46}$. It was further shown that this universality in vibrational spectrum held true also for protein capsids ${ }^{35}$. Second, it was shown in one of our recent work that a direct corollary of the universality in vibrational spectrum among globular proteins is that their magnitudes of thermal vibrations are nearly universal, having a narrow distribution that peaks at $0.093 \AA^{2}$ at $100 \mathrm{~K}^{31}$. In obtaining this value, a harmonic potential is assumed. 
The harmonicity assumption is reasonable for thermal vibrations at or below $100 \mathrm{~K}$. At higher temperature, particularly at above the glass transition temperature of proteins which is about 200-220 K, proteins undergo "glass transition" and become much more flexible ${ }^{47}$. As a result, the magnitude of mean-square displacements grows super-linearly. Therefore, the magnitude of thermal vibrations of globular proteins at $300 \mathrm{~K}$ is at least three times as high as $0.093 \AA^{2}$, or $0.279 \AA^{2}$. In other words, the magnitude of thermal vibrations computed from sbNMA (which assumes harmonicity) represents a lower bound of the actual magnitude at room temperature.

On the other hand, the magnitudes of thermal fluctuations of globular proteins are available in crystallographic B-factors, though in which unfortunately a large amount of static disorder co-resides. Because of static disorder, B-factors do not represent the actual magnitudes of thermal fluctuations but rather an upper bound.

The aforementioned upper and lower bounds thus define a range of the actual magnitudes of thermal vibrations of globular proteins. The range, though not as ideal as a single definite value, gives us a good sense of the magnitude.

Fig. 4 shows the upper and lower bounds of the magnitude of thermal fluctuations of the 18 structures in the dataset. For three out of the 18 structures, the lower bound obtained from the sbNMA computation is slightly higher than the upper bound obtained from Bfactors. This could be due to the imperfection of sbNMA or uncertainties in B-factors, or both. However, for most proteins, the over trend is that the upper bound is about 1.6-1.7 times higher than the lower bound. The mean lower and upper bounds of the proteins shown in Fig. 4 are 0.30 and $0.51 \AA^{2}$, respectively. 


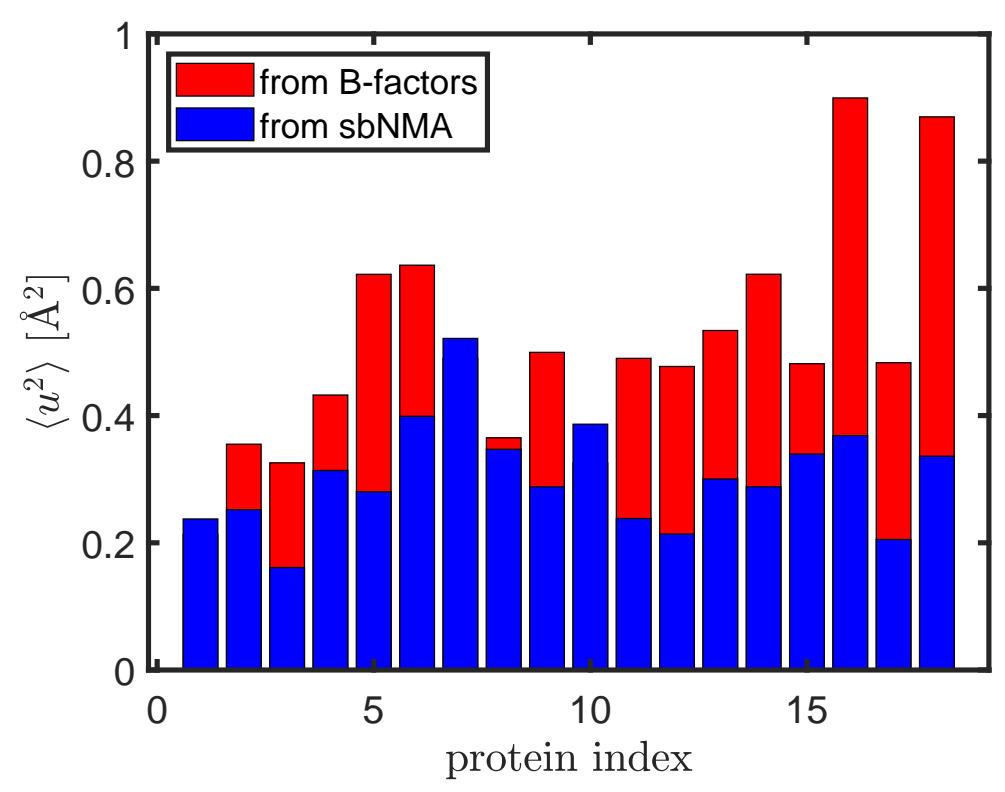

Figure 4: The upper and lower bounds of the magnitudes of thermal fluctuations of the 18 structures determined at room temperature and high resolution (1.1 $\AA$ or higher). For three out of the 18 structures, the lower bound is slightly higher than the upper bound.

\subsection{Glass Transition}

It is known that proteins undergo glass transition at $T_{g}$, the glass transition temperature, which is about $200-220 \mathrm{~K}$ for proteins ${ }^{37}$. At above $T_{g}$, proteins become much more flexible. Past work indicated that "the glass transition in hydrated samples is located in the surface layer of proteins and related to the (im)mobilization of the protein groups and strongly bound water." 4 . As a result, the magnitude of mean-square displacements grows super-linearly with temperature. 


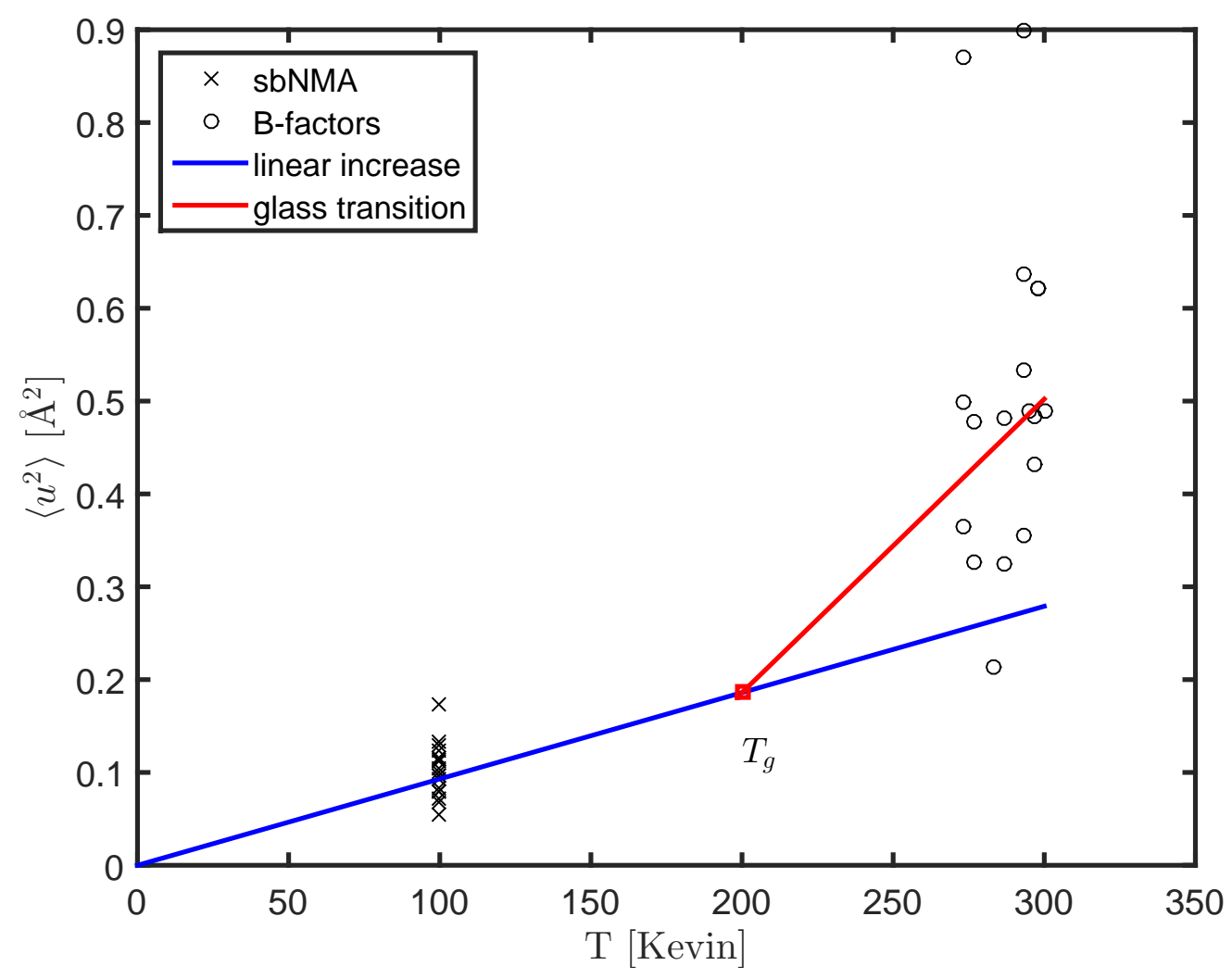

Figure 5: The magnitude of thermal fluctuations as a function of temperature. $T_{g}$ denotes the temperature of glass transition that is $200-220 \mathrm{~K}^{48}$. The magnitudes computed from sbNMA at 100K are marked by black crosses, while the magnitudes obtained from B-factors by black circles. The blue line represents the universal trend of the magnitude of harmonic vibrations by sbNMA with CHARMM force field, having a nearly universal value around $0.093 \AA^{2}$ at $100 \mathrm{~K}$ for all proteins ${ }^{31}$, and $0.279 \AA^{2}$ at $300 \mathrm{~K}$. At above $T_{g}$, anharmonic motions kick in and the magnitude of total thermal fluctuations increases superlinearly, as marked by the red line that is fitted to the black circles.

Fig. 5 presents the magnitudes of thermal vibrations as computed from sbNMA ${ }^{31,32}$ of the 18 proteins in the dataset, as well as their mean-square displacements from crystallographic B-factors. The blue line represents the linear growth of mean-square displacements as a function of temperature should there be no glass transition. The red line in Fig. 5 represents a fitting to $\left\langle u^{2}\right\rangle$ (of B-factors). A clear picture of glass transition emerges. 


\subsection{The range of Young's modulus}

Fig. 6 shows the range of Young's moduli computed from $\sigma$ ESM and calibrated with the magnitudes of thermal vibrations from sbNMA or B-factors. The median Young's modulus when fitting to B-factors is $6.03 \mathrm{Gpa}$, and 10.6 Gpa when fitting to sbNMA. sbNMA does not consider glass transition but only harmonic motions. Thus, the Young's moduli calibrated with sbNMA can be thought of as the Young's moduli of proteins at temperatures below $T_{g}$, while the Young's moduli fit to B-factors can be thought of as Young's moduli at the room temperature.

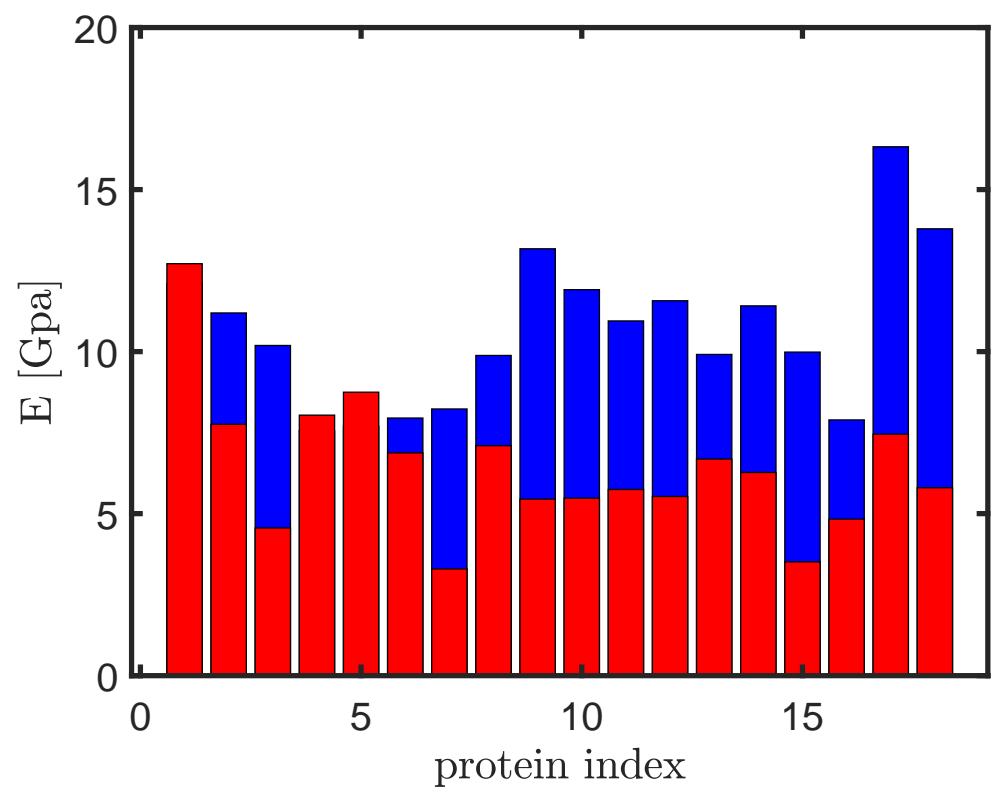

Figure 6: A bar plot shows the Young's moduli $(E)$ calibrated with sbNMA (in blue) and those with crystallographic B-factors (in red), for the 18 proteins in the dataset. The blue bars can be interpreted also the Young's moduli at temperatures below $T_{g}$, while the red bars Young's moduli at the room temperature. 


\subsection{Estimating the Young's modulus of the interface region using sbNMA and CHARMM force field}

The interface region is kept together mostly by van der Waals interactions. The van der Waals potential employed in the CHARMM force field is a 6-12 Leonard-Jones potential and takes the following form:

$$
V_{\mathrm{vdW}}=\epsilon\left(\left(\frac{r_{0}}{r}\right)^{12}-2\left(\frac{r_{0}}{r}\right)^{6}\right)
$$

The spring constant due to the van der Waals potential is:

$$
k_{\mathrm{vdW}}=\frac{\partial^{2} V_{\mathrm{vdW}}}{\partial r^{2}}=\frac{12 \epsilon}{r^{2}}\left(13\left(\frac{r_{0}}{r}\right)^{12}-7\left(\frac{r_{0}}{r}\right)^{6}\right)=\frac{72 \epsilon}{r_{0}^{2}}
$$

the last step of which assumes $r=r_{0}$.

Fig. 7 shows the histogram distribution of the van der Waals parameter $\epsilon$ of the 50 CHARMM atom types in par_all36_prot.prm and the histogram distribution of $k_{v d W}\left(r_{0}\right)$, which falls mostly in the range of 0.1 to $1 \mathrm{Kcal} / \mathrm{mol} / \AA^{2}$. It is thus clear that $k_{v d W}$, the spring constant due to van der Waals interactions, is much weaker than the spring constants for covalent bonds that are in the order of several hundreds $\mathrm{Kcal} / \mathrm{mol} / \AA^{2}$. 


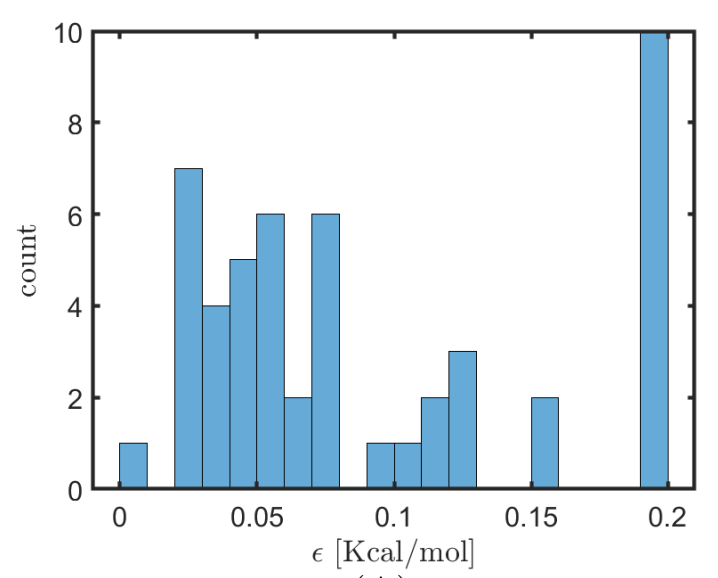

(A)

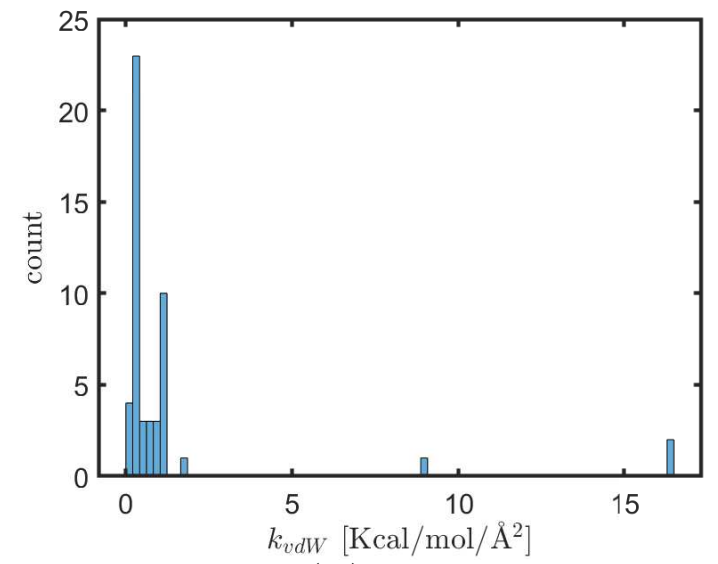

(B)

Figure 7: Histogram distributions of (A) the van der Waals parameter $\epsilon$ used in CHARMM and (B) of $k_{v d W}\left(r_{0}\right)$, the spring constant of van der Waal interactions.

Since the equilibrium distance between a pair of atoms are in the range of 2.5 to $4.5 \AA$, The Young's modulus of the interface region, $E_{\text {inter }}$, is estimated to be on the order of $\frac{k_{v d W}}{r_{0}}$, or 0.02 to $0.4 \mathrm{Kcal} / \mathrm{mol} / \AA^{3}$ (or 0.1 to $3 \mathrm{Gpa}$, the converting factor between the two units is 6.9). This is 2 to 3 orders smaller than that of covalent solids or ionic solids, which is expected since spring constants derived from van der Waals interactions are 2 to 3 orders smaller than those of covalent bonds or ionic bonds.

\subsection{Young's moduli of the protein interface regions}

Using the approach described in the Methods, we first generate quaternary structures of all the 18 proteins in the dataset. $\sigma$ ESM (see Methods section for details) is then applied to compute the Young's moduli of the interface regions (the MATLAB scripts used for this computation are available at https://github.com/gsongISU/sigmaESMrelease). The results are given in Table 2. It is seen that while the Young's moduli of the proteins are a few Gpa, the Young's moduli of interface regions are about an order smaller. Such a significant difference in stiffness between proteins and protein interfaces must be due to the 
underlying chain connectivity of proteins and the associated bonded interactions. Proteins can be considered as van der Waals solids with "steel enforcement" ${ }^{49}$. Also listed in Table 2 are the volumes of interface regions of the quaternary structures. In $\sigma$ ESM solid model, the interface region (the blue region in Fig. 3) is represented by a collection of tetrahedra elements. The volume of an interface region is defined as the total volume of all tetrahedra elements in the region.

Table 2: The lower and upper bounds of Young's moduli of the 18 proteins and the Young's moduli of the interface regions of the corresponding quaternary structures. The lower bounds are obtained by fitting $\sigma$ ESM results to B-factors, while the higher bounds are obtained by fitting the $\sigma$ ESM results to sbNMA. Also listed in the table are the sizes of the 18 proteins and the volumes of their interface regions. The unit for all Young's moduli is Gpa.

\begin{tabular}{|l||c|c||c|c||c|}
\hline pdb-id & residue \# & volume $\left[\mathrm{nm}^{3}\right]$ & $E_{\text {intra }}$ (lower bound) & $E_{\text {intra }}$ (upper bound) & $E_{\text {inter }}$ \\
\hline 1p9g & 41 & 0.89 & 12.71 & 12.10 & 0.09 \\
1rb9 & 52 & 1.04 & 7.76 & 11.19 & 0.58 \\
1iro & 53 & 1.72 & 4.56 & 10.19 & 0.56 \\
2igd & 61 & 1.42 & 8.04 & 7.56 & 0.40 \\
1aho & 64 & 1.62 & 8.75 & 7.70 & 1.17 \\
5tog & 75 & 2.14 & 6.88 & 7.95 & 0.56 \\
1ctj & 89 & 1.40 & 3.29 & 8.23 & 0.96 \\
1lwb & 122 & 1.60 & 7.10 & 9.88 & 0.58 \\
1c7k & 132 & 1.04 & 5.45 & 13.17 & 0.36 \\
3r87 & 132 & 3.76 & 5.48 & 11.91 & 0.83 \\
6gz8 & 133 & 1.47 & 5.75 & 10.95 & 1.13 \\
5vg0 & 142 & 1.72 & 5.53 & 11.57 & 0.23 \\
4b9g & 146 & 2.34 & 6.69 & 11.41 & 1.02 \\
4ann & 176 & 2.58 & 6.27 & 9.99 & 0.57 \\
4qa8 & 210 & 1.54 & 3.51 & 7.89 & 0.91 \\
6h40 & 220 & 1.52 & 4.83 & 16.32 & 0.57 \\
2pwa & 279 & 1.52 & 7.45 & 13.79 & 0.44 \\
6gy5 & 285 & 0.82 & 5.80 & 10.57 & 0.15 \\
\hline median & 132 & 1.53 & 6.03 & 0.57 \\
\hline
\end{tabular}

Another observation is that the Young's modulus of the interface region correlates somewhat with its volume. Fig. 8 shows a scatter plot of the volumes of interface regions of the 18 quaternary structures and the Young's moduli of their interface regions. A moderate correlation is seen (correlation coefficient: 0.38). 


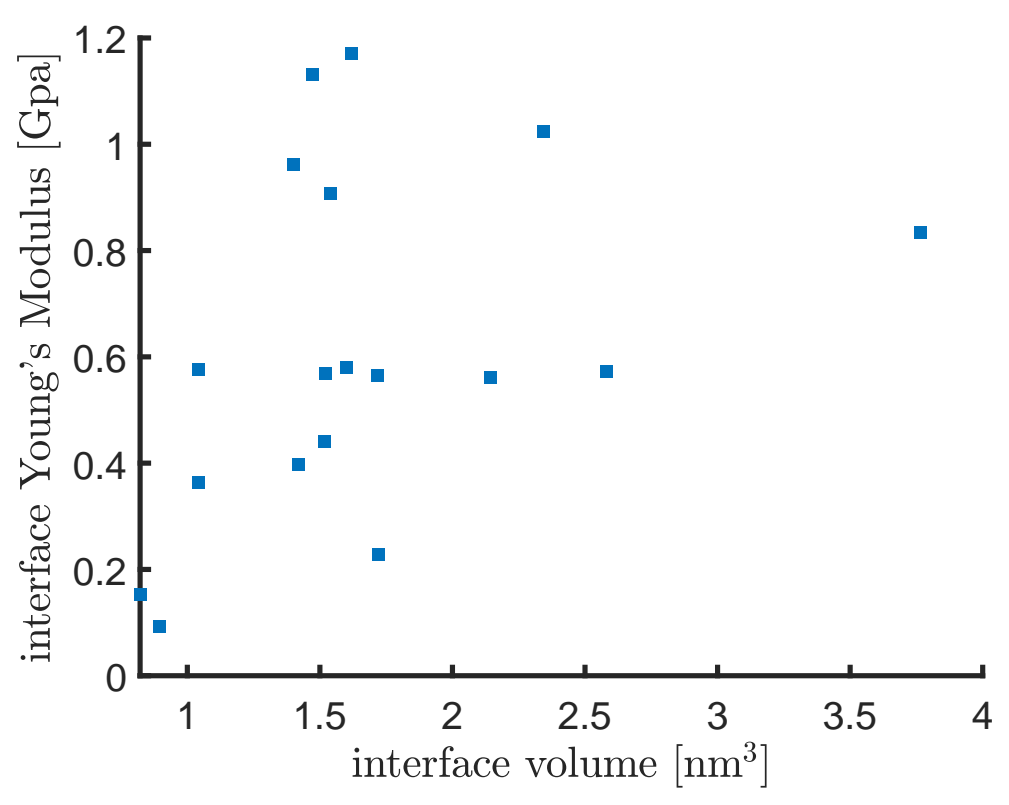

Figure 8: A scatter plot of the volumes of interface regions of the 18 quaternary sturctures and the Young's moduli of the interface regions.

\section{Discussion}

In this work, we have studied the elastic moduli of proteins and protein interfaces using a bottom-up approach. The material properties of solids in general are determined by the underlying physical interactions. For proteins and protein interfaces in particular, their material properties such as elasticity are dictated by the underlying molecular interactions.

The innovation of this work is the development of a novel method that can bridge between material properties at the macroscopic level and molecular interactions at the microscopic level. To achieve this, we employ both an all-atom NMA model (here sbNMA ${ }^{32}$ ) and an elastic solid model ( $\sigma$ ESM) for proteins and protein interfaces. And the "bridge" between the two models is a common physical property that they both can predict: the magnitude 
of thermal vibrations (see Eq. (7)), i.e.,

$$
\left\langle\Delta \mathbf{R}^{2}\right\rangle_{\mathrm{ESM}}=\left\langle\Delta \mathbf{R}^{2}\right\rangle_{\mathrm{NMA}}
$$

This connection allows us to determine the Young's moduli of proteins and protein interfaces molecular interactions.

A similar bridge can be built between $\sigma \mathrm{ESM}$ and crystallographic B-factors (Eq. 8) as well. A significant benefit of doing both is that the two constraints are complimentary to each other and together they provide a nice upper and lower bounds for the Young's moluli of proteins.

Atoms at the interface region interact primarily through van der Waals interactions. One key realization is that the spring constant due to the van der Waals interaction, or $k_{v d W}$, is small and is mostly in the range of $0.1-1 \mathrm{kcal} / \mathrm{mol} / \AA^{2}$ (Fig. 7). Since the separation distances $r_{0}$ between pairs of atoms at the interface region should be about the sum of their van der Waals radii, one may estimate the order of Young's modulus at the interface region by simply taking the ratio of the two, which comes to be about 0.1 to 3 Gpa. Our actual computations using the 18 proteins in the dataset are in agreement with this estimation. Covalent solids such as diamond have a Young's modulus that is hundreds of Gpa or even over 1,000 Gpa, which is about 3 orders higher than that of proteins or protein interface. The difference has a simple physical explanation: the spring constants for covalent bonds (as in CHARMM force field) are about 3 orders higher than the van der Waals spring constants while the separation distances between interacting atoms are similar. In a nutshell, the low Young's modulus at protein interface is directly due to the weak van der Waals interactions.

Another interesting finding is that, compared with the Young's modulus at the interface regions, the Young's moduli of proteins are one order higher, though still significantly softer than covalent solids. The latter is understandable as earlier work by Howard ${ }^{2}$ showed that 
the majority of interactions inside a protein were van der Waals interactions. Proteins are mostly van der Waals solids. On the other hand, proteins themselves are firmer than the interface regions. After all, proteins are chains of amino acids linked together by covalent bonds. The covalent chain serves as a "steel enforcement" 49 that stiffens a protein solid. The abundance of secondary structures in proteins provides additional enforcement through hydrogen bonds. As a result, the internal of proteins is about 10 times stiffer than the interface region.

As part of future work, we plan to apply the method to study the elastic properties of protein capsids. The stiffness of viral capsids has been measured for a large number of viruses using AFM technique ${ }^{5}$. The stiffness varies greatly among the viral capsids, with that of some capsids being an order of magnitude higher than that of others ${ }^{5}$. The physical cause of this large degree of variations in stiffness is not known. As mentioned in the Introduction, existing work, using with either the thin shell or thick shell models, all represents a top-down approach that uses experimental determined stiffness values to fit the underlying elastic moduli of capsids and were not able to provide a physical explanation for the observed stiffness. Our present approach has the potential to take into the account not only the structural details of protein capsids (instead of a shell with uniform spherical geometry and thickness), but also the striking difference in elastic properties between protein subunits and protein interface regions, and consequently may be used to predict capsid stiffness and provide an explanation for the observed differences in stiffness among viral capsids.

\section{References}

[1] M. Grodzicki. The concept of the chemical bond in solids. In Z. Maksić, editor, Theoretical Models of Chemical Bonding, Part 2: The Concept of the Chemical Bond, pages 417-452. Springer-Verlag, 1990. 
[2] Jonathon Howard. Mechanics of motor proteins and the cytoskeleton. Sinauer Associates Publishers, Sunderland, Mass., 2001.

[3] M. Guthold, W. Liu, E. A. Sparks, L. M. Jawerth, L. Peng, M. Falvo, R. Superfine, R. R. Hantgan, and S. T. Lord. A comparison of the mechanical and structural properties of fibrin fibers with other protein fibers. Cell Biochem Biophys, 49(3):165-81, 2007.

[4] V. N. Morozov and S. G. Gevorkian. Low-temperature glass-transition in proteins. Biopolymers, 24(9):1785-1799, 1985.

[5] M. G. Mateu. Mechanical properties of viruses analyzed by atomic force microscopy: a virological perspective. Virus Res, 168(1-2):1-22, 2012.

[6] H. Kojima, A. Ishijima, and T. Yanagida. Direct measurement of stiffness of single actinfilaments with and without tropomyosin by in-vitro nanomanipulation. Proceedings of the National Academy of Sciences of the United States of America, 91(26):12962-12966, 1994.

[7] R. D. Fraser and T. P. Macrae. Molecular structure and mechanical properties of keratins. Symp Soc Exp Biol, 34:211-46, 1980.

[8] J. Gilbert, M. Charnley, C. Cheng, N. P. Reynolds, and O. G. Jones. Quantifying young's moduli of protein fibrils and particles with bimodal force spectroscopy. Biointerphases, 12(4):041001, 2017.

[9] M. Guthold, W. Liu, B. Stephens, S. T. Lord, R. R. Hantgan, D. A. Erie, Jr. Taylor, R. M., and R. Superfine. Visualization and mechanical manipulations of individual fibrin fibers suggest that fiber cross section has fractal dimension 1.3. Biophys J, 87(6):4226$36,2004$. 
[10] B. Gavish, E. Gratton, and C. J. Hardy. Adiabatic compressibility of globular proteins. Proc Natl Acad Sci U S A, 80(3):750-4, 1983.

[11] C. E. Kundrot and F. M. Richards. Crystal-structure of hen egg-white lysozyme at a hydrostatic-pressure of 1000 atmospheres. Journal of Molecular Biology, 193(1):157$170,1987$.

[12] V. N. Morozov and T. Y. Morozova. Viscoelastic properties of protein crystals - triclinic crystals of hen egg-white lysozyme in different conditions. Biopolymers, 20(3):451-467, 1981.

[13] V. N. Morozov, T. Y. Morozova, G. S. Kachalova, and E. T. Myachin. Interpretation of water desorption isotherms of lysozyme. International Journal of Biological Macromolecules, 10(6):329-336, 1988.

[14] T. A. Zenchenko, E. V. Pozharskii, and V. N. Morozov. A magnetic micromethod to measure young's modulus of protein crystals and other polymer materials. J Biochem Biophys Methods, 33(3):207-15, 1996.

[15] S. Tait, E. T. White, and J. D. Litster. Mechanical characterization of protein crystals. Particle Particle Systems Characterization, 25(3):266-276, 2008.

[16] H. Koizumi, M. Tachibana, and K. Kojima. Elastic constants in tetragonal hen eggwhite lysozyme crystals containing large amount of water. Phys Rev E Stat Nonlin Soft Matter Phys, 79(6 Pt 1):061917, 2009.

[17] N. Kitajima, S. Tsukashima, D. Fujii, M. Tachibana, H. Koizumi, K. Wako, and K. Kojima. Elastic constants in orthorhombic hen egg-white lysozyme crystals. Phys Rev E Stat Nonlin Soft Matter Phys, 89(1):012714, 2014. 
[18] S. Speziale, F. Jiang, C. L. Caylor, S. Kriminski, C. S. Zha, R. E. Thorne, and T. S. Duffy. Sound velocity and elasticity of tetragonal lysozyme crystals by brillouin spectroscopy. Biophys J, 85(5):3202-13, 2003.

[19] J. P. Michel, I. L. Ivanovska, M. M. Gibbons, W. S. Klug, C. M. Knobler, G. J. Wuite, and C. F. Schmidt. Nanoindentation studies of full and empty viral capsids and the effects of capsid protein mutations on elasticity and strength. Proc Natl Acad Sci US A, 103(16):6184-9, 2006.

[20] M. M. Gibbons and W. S. Klug. Influence of nonuniform geometry on nanoindentation of viral capsids. Biophysical Journal, 95(8):3640-3649, 2008.

[21] M. Bathe. A finite element framework for computation of protein normal modes and mechanical response. Proteins, 70(4):1595-609, 2008.

[22] N. Go, T. Noguti, and T. Nishikawa. Dynamics of a small globular protein in terms of low-frequency vibrational modes. Proc. Natl. Acad. Sci. USA, 80(12):3696-3700, June 1983.

[23] M. Levitt, C. Sander, and P. S. Stern. The normal modes of a protein: Native bovine pancreatic trypsin inhibitor. Int. J. Quant. Chem., 10:181-199, 1983.

[24] B. Brooks and M. Karplus. Harmonic dynamics of proteins: normal modes and fluctuations in bovine pancreatic trypsin inhibitor. Proc. Natl. Acad. Sci. USA, 80(21):65716575, November 1983.

[25] G. Song. Shape-preserving elastic solid models of macromolecules. Plos Computational Biology, 16(5), 2020.

[26] H. Edelsbrunner and E. P. Mucke. Three-dimensional alpha shapes. ACM Transactions on Graphics, 13(1):43-72, 1994. 
[27] M. F. Sanner, A. J. Olson, and J. C. Spehner. Reduced surface: an efficient way to compute molecular surfaces. Biopolymers, 38(3):305-20, 1996.

[28] János Inczédy, Tamás Lengyel, Allan M. Ure, and András Gelencsér. Compendium of analytical nomenclature: definitive rules 199\%. Blackwell Science, Oxford, 3 edition, 1998.

[29] T. Rahman and J. Valdman. Fast matlab assembly of fem matrices in 2d and 3d: Nodal elements. Applied Mathematics and Computation, 219(13):7151-7158, 2013.

[30] Daryl L. Logan. A first course in the finite element method. Thomson, Toronto, Canada, 4th edition, 2007.

[31] H. Na, K. Hinsen, and G. Song. The amounts of thermal vibrations and static disorder in protein x-ray crystallographic b-factors. Authorea, 2020.

[32] H. Na and G. Song. Bridging between normal mode analysis and elastic network models. Proteins, 82:2157-2168, September 2014.

[33] A. D. MacKerell, D. Bashford, Bellott, R. L. Dunbrack, J. D. Evanseck, M. J. Field, S. Fischer, J. Gao, H. Guo, S. Ha, D. Joseph-McCarthy, L. Kuchnir, K. Kuczera, F. T. K. Lau, C. Mattos, S. Michnick, T. Ngo, D. T. Nguyen, B. Prodhom, W. E. Reiher, B. Roux, M. Schlenkrich, J. C. Smith, R. Stote, J. Straub, M. Watanabe, J. WiórkiewiczKuczera, D. Yin, and M. Karplus. All-atom empirical potential for molecular modeling and dynamics studies of proteins. J. Phys. Chem. B, 102(18):3586-3616, April 1998.

[34] H. Na, G. Song, and D. ben Avraham. Universality of vibrational spectra of globular proteins. Phys Biol, 13(1):016008, 2016.

[35] H. Na and G. Song. Fast normal mode computations of capsid dynamics inspired by resonance. Phys Biol, 15(4):046003, 2018. 
[36] H. Na and G. Song. Conventional nma as a better standard for evaluating elastic network models. Proteins, 83(2):259-67, 2015.

[37] D. Ringe and G. A. Petsko. The 'glass transition' in protein dynamics: what it is, why it occurs, and how to exploit it. Biophys. Chem., 105(2-3):667-80, 2003.

[38] E. Fuglebakk, N. Reuter, and K. Hinsen. Evaluation of protein elastic network models based on an analysis of collective motions. J Chem Theory Comput, 9(12):5618-28, 2013.

[39] C. J. Burden and A. J. Oakley. Anisotropic atomic motions in high-resolution protein crystallography molecular dynamics simulations. Phys Biol, 4(2):79-90, 2007.

[40] R. A. Sayle and E. J. Milnerwhite. Rasmol - biomolecular graphics for all. Trends in Biochemical Sciences, 20(9):374-376, 1995.

[41] W. Humphrey, A. Dalke, and K. Schulten. VMD - Visual Molecular Dynamics. J Mol. Graphics, 14:33-38, 1996.

[42] F. Tama, F.X. Gadea, O. Marques, and Y.H. Sanejouand. Building-block approach for determining low-frequency normal modes of macromolecules. Proteins, 41(1):1-7, 2000.

[43] David W. Rodgers. Cryocrystallography. Structure, 2(12):1135-1140, Dec 1994.

[44] R. W. W. Hooft, C. Sander, and G. Vriend. Verification of protein structures: Side-chain planarity. Journal of Applied Crystallography, 29:714-716, 1996.

[45] W. G. Touw, C. Baakman, J. Black, T. A. H. te Beek, E. Krieger, R. P. Joosten, and G. Vriend. A series of pdb-related databanks for everyday needs. Nucleic Acids Research, 43(D1):D364-D368, 2015. 
[46] D. ben-Avraham. Vibrational normal-mode spectrum of globular proteins. Phys. Rev. $B, 47(21): 14559-60,1993$.

[47] Dagmar Ringe and Gregory A. Petsko. The 'glass transition' in protein dynamics: What it is, why it occurs, and how to exploit it. Biophysical Chemistry, 105(2-3):667-680, September 2003.

[48] D. Ringe and G. A. Petsko. The 'glass transition' in protein dynamics: what it is, why it occurs, and how to exploit it. Biophys Chem, 105(2-3):667-80, 2003.

[49] K. Hinsen. The stuff that proteins are made of: physical properties of folded peptide chains, 2017. A research presentation, available at https://www.youtube.com/watch?v=RbOu1Vjp3Mc. 